\title{
Dynamic buffer capacity versus alkalinity. Formulation in terms of Simms constants idea
}

Received: 08 January, 2020

Accepted: 10 February, 2020

Published: 12 February, 2020

*Corresponding author: Tadeusz Michałowski, Department of Analytical Chemistry, Technical University of Cracow, Cracow, Poland, E-mail: michalot@o2.pl

Keywords: Acid-base equilibria; Simms constants; Titration curves; Fulvic acids; Metal complexation; Total alkalinity; Natural waters; Lymphatic systems

https://www. peertechz.com

Check for updates

\author{
Anna Maria Michałowska-Kaczmarczyk ${ }^{1}$ and Tadeusz \\ Michałowski2* \\ 'Department of Oncology, The University Hospital in Cracow, Cracow, Poland \\ ${ }^{2}$ Department of Analytical Chemistry, Technical University of Cracow, Cracow, Poland
}

\begin{abstract}
The Simms constants $\left(g_{i}\right)$ concept is put in context with equations for some acid-base titration curves, formulated for $D+T$ systems, with titrand $D$ and titrant $T$ prepared according to normal and isomolar mode. The relationships between $\mathrm{g}_{\mathrm{i}}$ and successive dissociation constants $(\mathrm{K})$ values for polyprotic acids are formulated. The mathematical formulation of dynamic buffer capacity concept is presented in general and elegant form, involving all soluble species formed in the system where only acid-base reactions are involved. The Simms constants are identified with protonation micro-constants related to specific basicity centers.
\end{abstract}

\section{Abbreviations}

C: Concentration [mol/L] of HB in T, D - titrand (solutions titrated), $\Delta=\mathrm{C}_{\mathrm{b}}-\mathrm{C}_{\mathrm{a}}, \mathrm{g}_{\mathrm{i}}-\mathrm{Simms}$ constant, $\mathrm{g}_{\mathrm{i}}^{*}=\gamma \cdot \mathrm{g}_{\mathrm{i}}-$ hybrid Simms constant, $\gamma$-activity coefficient of $\mathrm{H}^{+1}$ ions, $\mathrm{h}=\gamma \cdot[\mathrm{H}]-$ activity of $\mathrm{H}^{+1}$ ions, HB - strong acid, MOH - strong base, ST - sample tested as: $\mathrm{s}$-ST $=$ simple sample tested or $\mathrm{c}-\mathrm{ST}=$ complex sample tested; $\mathrm{T}$ - titrant, $\mathrm{V}$ - volume [mL] of $\mathrm{T}, \mathrm{V}_{0}$ - volume $[\mathrm{mL}]$ of $\mathrm{D}, \mathrm{W}=\mathrm{V}_{0}+\mathrm{V},\left[\mathrm{X}_{\mathrm{i}}\right]$ concentration $[\mathrm{mol} / \mathrm{L}]$ of the species $\mathrm{X}_{\mathrm{i}}^{\mathrm{z}_{\mathrm{i}}}$.

\section{Introduction}

The term 'Simms constants' is an eponym for virtual equilibrium constants $\left(\mathrm{g}_{\mathrm{i}}\right)$ suggested by Simms $[1,2]$ and presented elsewhere only as an alternative mathematical formalism applied to simple static acidbase systems. The Simms constants idea was extended $[3,4]$, on modeling of acid-base titration realized according to isomolar mode [5-11]. Its further extension concerned the systems where total alkalinity (TAL) was modelled $[12,13]$, also in the systems with fulvic acids [14]. This idea can be extended on biological systems, such as the lymphatic system in living organisms. All the new possibilities offered by Simms constants idea were suggested by Michałowski [15].

The Simms constants idea resembles the mathematical problem known from integral calculus as partial fraction decomposition [16], related to some rational functions $\mathrm{R}(\mathrm{x})=\mathrm{f}(\mathrm{x}) / \mathrm{g}(\mathrm{x})$, where: $a$. the numerator $\mathrm{f}(\mathrm{x})$ and the denominator $\mathrm{g}(\mathrm{x})$ are both polynomials of variable $x$; $b$. the degree of $f(x)$ is smaller than that of the $\mathrm{g}(\mathrm{x})$; c. $\mathrm{g}(\mathrm{x})$ is irreducible polynomial [17], i.e., it cannot be expressed as the product of two polynomials.

Application of the Simms constants $g_{i}$ enables any q-protic acid $\mathrm{H}_{\mathrm{n}} \mathrm{L}\left(\mathrm{C}_{0} \mathrm{~mol} / \mathrm{L}\right)$, characterized by successive dissociation constants $\mathrm{K}_{\mathrm{i}}$ values, to be considered as a mixture of q monoprotic acids $\mathrm{HL}_{(\mathrm{i})}$ $(\mathrm{i}=1, \ldots, \mathrm{q})$ of the same concentration, i.e. $\mathrm{C}_{0} \mathrm{~mol} / \mathrm{L}$; the $\mathrm{g}_{\mathrm{i}}$ are ascribed to these acids as (virtual) dissociation 
constants values. The relations between $g_{i}$ and $K_{i}$ values were formulated [14].

This idea will be presented below starting from equations for acid-base titration curves. In a titration $\mathrm{T}(\mathrm{V}) \Rightarrow \mathrm{D}\left(\mathrm{V}_{0}\right), \mathrm{V}_{0} \mathrm{~mL}$ of titrand (D) is titrated with $\mathrm{V}$ $\mathrm{mL}$ of titrant $(\mathrm{T})$ and $\mathrm{V}_{0}+\mathrm{V} \mathrm{mL}$ of $\mathrm{D}+\mathrm{T}$ mixture is obtained, if the additivity of $\mathrm{V}_{0}$ and $\mathrm{V}$ is assumed. We apply here the abbreviated notation, where charges $\mathrm{z}_{\mathrm{i}}$ of $\mathrm{i}$-th species $\mathrm{X}_{\mathrm{i}}^{\left(\mathrm{z}_{\mathrm{i}}\right)}$ be omitted when written in terms of molar concentration, $\left[\mathrm{X}_{\mathrm{i}}\right]$.

Titrations in normal and isomolar D+T systems will be formulated. In the normal mode, a sample tested (ST) as titrand D is titrated with HB (C) solution as titrant T. In isomolar systems, ST is a component of $\mathrm{D}$ and $\mathrm{T}$, prepared as presented in Table 1. Simple (s-) and complex (c-) acid-base systems are considered herein.

\section{Titrations in simple D+T systems}

The principle of the Simms constants formulation will be illustrated first for the $\mathrm{M}_{\mathrm{k}} \mathrm{H}_{\mathrm{n}-\mathrm{k}} \mathrm{L}\left(\mathrm{C}_{0}\right)+\mathrm{HB}\left(\mathrm{C}_{\mathrm{a}}\right)+$ $\mathrm{MOH}\left(\mathrm{C}_{\mathrm{b}}\right)$ mixture, considered here as the simple sample tested (s-ST) in the systems I and II.

\section{Simple D+T system (I) - normal mode}

Let $\mathrm{V}_{0} \mathrm{~mL}$ of the s-ST, considered here as $\mathrm{D}$, be titrated with $\mathrm{V}$ mL of a strong acid HB (C) as T. The charge and concentration balances are as follows :

$$
\alpha+[\mathrm{M}]-[\mathrm{B}]+\sum_{\mathrm{i}=1}^{\mathrm{q}}(\mathrm{i}-\mathrm{n})\left[\mathrm{H}_{\mathrm{i}} \mathrm{L}\right]=0
$$

$[\mathrm{M}]=\left(\mathrm{kC}_{0}+\mathrm{C}_{\mathrm{b}}\right) \mathrm{V}_{0} / \mathrm{W}$,

$[\mathrm{B}]=\left(\mathrm{C}_{\mathrm{a}} \mathrm{V}_{0}+\mathrm{CV}\right) / \mathrm{W}$,

$\sum_{\mathrm{i}=1}^{\mathrm{q}}\left[\mathrm{H}_{\mathrm{i}} \mathrm{L}\right]=\mathrm{C}_{0} \mathrm{~V}_{0} / \mathrm{W}$

where: $\mathrm{W}=\mathrm{V}_{0}+\mathrm{V}$ is the current volume of the $\mathrm{D}+\mathrm{T}$ mixture,

$$
\begin{aligned}
& \alpha=[H]-[O H]=[H]-K_{w} /[H]=h / \gamma-K_{w} \cdot \gamma / h \\
& h=\gamma \cdot[H], \quad p h=-\log h
\end{aligned}
$$

$\mathrm{h}$ is the activity and $\gamma-$ the activity coefficient of $\mathrm{H}^{+1}$ ions with concentration $[\mathrm{mol} / \mathrm{L}]$ denoted as $[\mathrm{H}]$. Let

$$
\overline{\mathrm{n}}^{(\mathrm{q})}=\frac{\sum_{\mathrm{i}=1}^{\mathrm{q}} \mathrm{i} \cdot\left[\mathrm{H}_{\mathrm{i}} \mathrm{L}\right]}{\sum_{\mathrm{i}=0}^{\mathrm{q}}\left[\mathrm{H}_{\mathrm{i}} \mathrm{L}\right]}=\frac{\sum_{\mathrm{i}=1}^{\mathrm{q}} \mathrm{i} \cdot \mathrm{K}_{\mathrm{i}}^{\mathrm{H}}[\mathrm{H}]^{\mathrm{i}}}{\sum_{\mathrm{i}=0}^{\mathrm{q}} \mathrm{K}_{\mathrm{i}}^{\mathrm{H}}[\mathrm{H}]^{\mathrm{i}}}
$$

be the mean number of protons attached to the basic form $\mathrm{L}^{-\mathrm{n}}$, and

$$
\mathrm{K}_{\mathrm{i}}^{\mathrm{H}}=\frac{\left[\mathrm{H}_{\mathrm{i}} \mathrm{L}\right]}{[\mathrm{H}]^{\mathrm{i}}[\mathrm{L}]} \text { for } \mathrm{iH}^{+1}+\mathrm{L}^{-\mathrm{n}}=\mathrm{H}_{\mathrm{i}} \mathrm{L}^{+\mathrm{i}-\mathrm{n}}
$$

$$
(\mathrm{i}=1, \ldots, \mathrm{q}) ; \mathrm{q}=\max \{\mathrm{i}\} ; \mathrm{K}_{0}^{\mathrm{H}} \equiv 1
$$

The $\mathrm{K}_{\mathrm{i}}^{\mathrm{H}}$ are interrelated with the dissociation constants $K_{j}(j=1, \ldots, q)$ values

$$
\mathrm{K}_{\mathrm{j}}=\frac{[\mathrm{H}]\left[\mathrm{H}_{\mathrm{q}-\mathrm{j}} \mathrm{L}\right]}{\left[\mathrm{H}_{\mathrm{q}-\mathrm{j}+1} \mathrm{~L}\right]} \text { for } \mathrm{H}_{\mathrm{q}-\mathrm{j}+1} \mathrm{~L}^{+\mathrm{q}-\mathrm{j}-\mathrm{n}}=\mathrm{H}_{\mathrm{q}-\mathrm{j}} \mathrm{L}^{+\mathrm{q}-\mathrm{j}-1-\mathrm{n}}+\mathrm{H}^{+1}
$$

in the relationships

$$
\mathrm{K}_{\mathrm{i}}^{\mathrm{H}}=\frac{1}{\prod_{\mathrm{j}=\mathrm{q}-\mathrm{i}+1}^{\mathrm{q}} \mathrm{K}_{\mathrm{j}}} \quad(\mathrm{i}=1, \ldots, \mathrm{q})
$$

From Equations 1, 2 and 5 we have, by turns,

$$
\begin{aligned}
& \sum_{\mathrm{i}=1}^{\mathrm{q}} \mathrm{i}\left[\mathrm{H}_{\mathrm{i}} \mathrm{L}\right]=\overline{\mathrm{n}}^{(\mathrm{q})} \cdot \mathrm{C}_{0} \mathrm{~V}_{0} / \mathrm{W} \\
& \alpha+\left(\mathrm{kC}_{0}+\mathrm{C}_{\mathrm{b}}\right) \mathrm{V}_{0} / \mathrm{W}-\left(\mathrm{C}_{\mathrm{a}} \mathrm{V}_{0}+\mathrm{CV}\right) / \mathrm{W}+ \\
& \left(\mathrm{n}-\overline{\mathrm{n}}^{(\mathrm{q})}\right) \mathrm{C}_{0} \mathrm{~V}_{0} / \mathrm{W}=0 \\
& \mathrm{CV} / \mathrm{W}=\alpha+\left(\left(\overline{\mathrm{n}}^{(\mathrm{q})}+\mathrm{k}-\mathrm{n}\right) \mathrm{C}_{0}+\Delta\right) \mathrm{V}_{0} / \mathrm{W}
\end{aligned}
$$

where $\Delta=\mathrm{C}_{\mathrm{b}}-\mathrm{C}_{\mathrm{a}}$. We apply the identity

$$
\bar{n}^{(q)}+k-n \equiv(q-n+k)-\left(q-\bar{n}^{(q)}\right)
$$

and notations:

$$
\begin{aligned}
& \mathrm{A}=(\mathrm{q}-\mathrm{n}+\mathrm{k}) \cdot \mathrm{C}_{0}+\Delta \\
& \mathrm{G}^{(\mathrm{q})}=\mathrm{q}-\overline{\mathrm{n}}^{(\mathrm{q})}=\frac{\sum_{\mathrm{i}=1}^{\mathrm{q}}(\mathrm{q}-\mathrm{i}) \cdot\left[\mathrm{H}_{\mathrm{i}} \mathrm{L}\right]}{\sum_{\mathrm{i}=0}^{\mathrm{q}}\left[\mathrm{H}_{\mathrm{i}} \mathrm{L}\right]} \\
& =\sum_{\mathrm{i}=1}^{\mathrm{q}} \frac{\mathrm{g}_{\mathrm{i}}}{[\mathrm{H}]+\mathrm{g}_{\mathrm{i}}}=\sum_{\mathrm{i}=1}^{\mathrm{q}} \frac{\mathrm{g}_{\mathrm{i}}^{*}}{\mathrm{~h}+\mathrm{g}_{\mathrm{i}}^{*}}
\end{aligned}
$$

where the Simms constants $\left(g_{i}\right)$ and hybrid Simms constants $\left(\mathrm{g}_{\mathrm{i}}^{*}\right)[3]$

$$
g_{i}^{*}=\gamma \cdot g_{i}, \quad \operatorname{pg}_{i}^{*}=-\operatorname{logg}_{i}^{*}(i=1, \ldots, q)
$$

are involved (see notation in Eq. 4). The A value (Eq. 8 ) is termed as alkalinity, perceived here as a simple case of total alkalinity, TAL [12-15]. Then from Eq. 6 we get, by turns,

$$
\begin{aligned}
& \mathrm{CV} / \mathrm{V}_{0}=\mathrm{A}+\alpha \cdot\left(1+\mathrm{V} / \mathrm{V}_{0}\right)-\mathrm{G}^{(\mathrm{q})} \cdot \mathrm{C}_{0} \\
& (\mathrm{C}-\alpha) \cdot \mathrm{V} / \mathrm{V}_{0}=\mathrm{A}-\mathrm{G}^{(\mathrm{q})} \cdot \mathrm{C}_{0}+\alpha \\
& \mathrm{V}=\mathrm{V}_{0} \cdot \frac{\mathrm{A}-\mathrm{G}^{(\mathrm{q})} \cdot \mathrm{C}_{0}+\alpha}{\mathrm{C}-\alpha}, \mathrm{W}=\mathrm{V}_{0} \cdot \frac{A-\mathrm{G}^{(\mathrm{q})} \cdot \mathrm{C}_{0}+\mathrm{C}}{\mathrm{C}-\alpha}
\end{aligned}
$$


Current concentration of $\mathrm{HB}$ from $\mathrm{T}$ in $\mathrm{D}+\mathrm{T}$ mixture is [18-20]

$$
\begin{aligned}
& c=C \cdot \frac{V}{W}=C \cdot \frac{A-G^{(q)} \cdot C_{0}+\alpha}{A-G^{(q)} \cdot C_{0}+C} \\
& =C \cdot\left(\frac{A-G^{(q)} \cdot C_{0}+C-(C-\alpha)}{A-G^{(q)} \cdot C_{0}+C}\right)=C \cdot\left(1-\frac{C-\alpha}{A+C-G^{(q)} \cdot C_{0}}\right)
\end{aligned}
$$

\section{Simple D+T system (II) - isomolar mode}

The condition for (approximately) constant ionic strength is fulfilled in isomolar D+T system [3,4], where $\mathrm{D}$ and $\mathrm{T}$ are prepared in identical flasks $\left(\mathrm{V}_{\mathrm{f}} \mathrm{mL}\right)$ according to the scheme illustrated in Table 1 . The isomolarity condition for the $\mathrm{D}+\mathrm{T}$ mixture during the $\mathrm{pH}$ titration is expressed by relation

$$
\mathrm{C}_{1} \mathrm{~V}_{\mathrm{MB}}=\mathrm{C}_{1} \mathrm{~V}_{\mathrm{MB}}^{*}+\mathrm{CV}_{\mathrm{B}}
$$

for the $\mathrm{D}+\mathrm{T}$ system with $\mathrm{HB}(\mathrm{C})$ in $\mathrm{T}$. From ionic strength viewpoint, the presence of $\mathrm{HB}(\mathrm{C})$ in $\mathrm{T}$ is compensated by a surplus $\mathrm{V}_{\mathrm{MB}}-\mathrm{V}_{\mathrm{MB}}^{*}=\mathrm{CV}_{\mathrm{B}} / \mathrm{C}_{1}$ of $\mathrm{MB}$ $\left(C_{1}\right)$ in the second flask, where $D$ is prepared. Both flasks are filled up to the marks $\left(\mathrm{V}_{\mathrm{f}}\right)$ with water. It is assumed that $\mathrm{C}_{1} \mathrm{~V}_{\mathrm{MB}} \gg \mathrm{CV}_{\mathrm{B}}$; then the isomolarity condition ensures also constancy of dielectric permittivity and improves the additivity of volumes $\mathrm{V}_{0}$ and $\mathrm{V}$.

In the system II, the charge balance (Eq. 1) and the relations:

$$
\begin{aligned}
& \sum_{\mathrm{i}=1}^{\mathrm{q}}\left[\mathrm{H}_{\mathrm{i}} \mathrm{L}\right]=\mathrm{d} \cdot \mathrm{C}_{0} \\
& \sum_{\mathrm{i}=1}^{\mathrm{q}} \mathrm{i}\left[\mathrm{H}_{\mathrm{i}} \mathrm{L}\right]=\overline{\mathrm{n}}^{(\mathrm{q})} \cdot \mathrm{d} \cdot \mathrm{C}_{0}
\end{aligned}
$$

are valid, where $d=\frac{v_{S T}}{V_{f}}$. From Equations $1,12-14$ we have

$$
\alpha+[M]-[B]+\left(\bar{n}^{(q)}-n\right) \cdot d \cdot C_{0}=0
$$

where:

$$
\begin{aligned}
& {[\mathrm{M}]=\mathrm{d} \cdot\left(\mathrm{k} \cdot \mathrm{C}_{0}+\mathrm{C}_{\mathrm{b}}\right)+\frac{\mathrm{C}_{1} \mathrm{~V}_{\mathrm{MB}}}{\mathrm{V}_{\mathrm{f}}}-\frac{\mathrm{CV}_{\mathrm{B}}}{\mathrm{V}_{\mathrm{f}}} \cdot \frac{\mathrm{V}}{\mathrm{W}} ;} \\
& {[\mathrm{B}]=\mathrm{d} \cdot \mathrm{C}_{\mathrm{a}}+\frac{\mathrm{C}_{1} \mathrm{~V}_{\mathrm{MB}}}{\mathrm{V}_{\mathrm{f}}}}
\end{aligned}
$$

Table 1: Composition of titrand (D) and titrant (T) for an isomolar $\mathrm{D}+\mathrm{T}$ system (II, IV); ST-sample tested (as S-ST or c-ST); HB-strong acid; $\mathrm{MOH}$-strong base;

$\mathrm{d}=\mathrm{V}_{\mathrm{ST}} / \mathrm{V}_{\mathrm{f}}$.

\begin{tabular}{|c|c|c|}
$\mathrm{d}=\mathrm{V}_{\mathrm{ST}} / \mathrm{V}_{\mathrm{f}}$. & \multicolumn{1}{|c|}{$\mathbf{T}$} \\
\hline Component & $\mathbf{D}$ & $\mathrm{V}_{\mathrm{ST}}$ \\
\hline $\mathrm{ST}$ & $\mathrm{V}_{\mathrm{ST}}$ & $\mathrm{V}_{\mathrm{B}}$ \\
\hline $\mathrm{HB}(\mathrm{C})$ & - & $\mathrm{V}_{\mathrm{MB}}^{*}$ \\
\hline $\mathrm{MB}\left(\mathrm{C}_{1}\right)$ & $\mathrm{V}_{\mathrm{MB}}$ & $\mathrm{V}_{\mathrm{f}}-\mathrm{V}_{\mathrm{ST}}-\mathrm{V}_{\mathrm{B}}-\mathrm{V}_{\mathrm{MB}}^{*}$ \\
\hline $\mathrm{H}_{2} \mathrm{O}$ & $\mathrm{V}_{\mathrm{f}}-\mathrm{V}_{\mathrm{ST}}-\mathrm{V}_{\mathrm{MB}}$ &
\end{tabular}

Putting Equations 7 and 16 in Eq. 15 gives

$$
\begin{aligned}
& \alpha+d \cdot\left(k \cdot C_{0}+C_{b}\right)-\frac{C V_{B}}{V_{f}} \cdot \frac{V}{W}-d \cdot C_{a}+ \\
& \left(\bar{n}^{(q)}-n\right) \cdot d \cdot C_{0}=0 \quad \Rightarrow \\
& \frac{\mathrm{CV}_{B}}{V_{f}} \cdot \frac{V}{W}=\left((q-n+k) \cdot C_{0}+\Delta\right) \cdot d+\alpha- \\
& \left(q-\bar{n}^{(q)}\right) \cdot d \cdot C_{0} \Rightarrow \\
& c=\frac{C V_{B}}{V_{f}} \cdot \frac{V}{W}=d \cdot A+\alpha-d \cdot G^{(q)} \cdot C_{0}
\end{aligned}
$$

where $A$ is expressed by Eq. 8. Note that $\mathrm{CV}_{B} / V_{f}=C_{B}$ is the concentration of $\mathrm{HB}$ in $\mathrm{T}$, prepared as indicated in Table 1, i.e. $\mathrm{c}=\mathrm{C}_{\mathrm{B}} \mathrm{V} / \mathrm{W}$ is the current concentration of HB from $T$ in the D+T mixture (compare with a remark at Eq. 11).

\section{Titrations in complex D+T systems}

In further parts of the paper, we consider a mixture

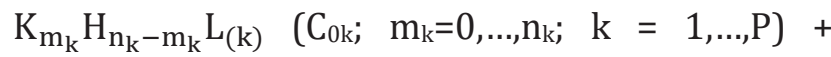

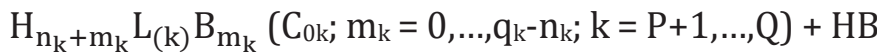
$\left(\mathrm{C}_{\mathrm{a}}\right)+\mathrm{MOH}\left(\mathrm{C}_{\mathrm{b}}\right)$ as the complex sample tested (c-ST).

\section{Complex D+T system (III) - normal mode}

$\mathrm{V}_{0} \mathrm{~mL}$ of the c-ST as D is titrated with $\mathrm{V}$ mL of $\mathrm{HB}$ (C) as T. We have the balances:

$$
\begin{aligned}
& \alpha+[\mathrm{K}]+[\mathrm{M}]-[\mathrm{B}]+\sum_{\mathrm{k}=1}^{\mathrm{Q}} \sum_{\mathrm{j}=0}^{\mathrm{q}_{\mathrm{k}}}\left(\mathrm{j}-\mathrm{n}_{\mathrm{k}}\right)\left[\mathrm{H}_{\mathrm{j}} \mathrm{L}_{(\mathrm{k})}\right]=0 \\
& \sum_{\mathrm{j}=0}^{\mathrm{q}_{\mathrm{k}}}\left[\mathrm{H}_{\mathrm{j}} \mathrm{L}_{(\mathrm{k})}\right]=\mathrm{C}_{0 \mathrm{k}} \cdot \frac{\mathrm{V}_{0}}{\mathrm{~W}} \\
& (\mathrm{k}=1, \ldots, \mathrm{P}, \mathrm{P}+1, \ldots, \mathrm{Q}) ;[\mathrm{K}]=\sum_{\mathrm{k}=1}^{\mathrm{P}} \mathrm{m}_{\mathrm{k}} \mathrm{C}_{0 \mathrm{k}} \cdot \frac{\mathrm{V}_{0}}{\mathrm{~W}} \\
& {[\mathrm{M}]=\mathrm{C}_{\mathrm{b}} \cdot \frac{\mathrm{V}_{0}}{\mathrm{~W}} ;} \\
& {[\mathrm{B}]=\left(\sum_{\mathrm{k}=\mathrm{P}+1}^{\mathrm{Q}} \mathrm{m}_{\mathrm{k}} \mathrm{C}_{0 \mathrm{k}}+\mathrm{C}_{\mathrm{a}}\right) \cdot \frac{\mathrm{V}_{0}}{\mathrm{~W}}+\mathrm{C} \cdot \frac{\mathrm{v}}{\mathrm{W}}}
\end{aligned}
$$


Applying Equations 19 and

$$
\begin{aligned}
& \sum_{\mathrm{j}=0}^{\mathrm{q}_{\mathrm{k}}} \mathrm{j} \cdot\left[\mathrm{H}_{\mathrm{j}} \mathrm{L}_{(\mathrm{k})}\right]=\overline{\mathrm{n}}_{\mathrm{k}}^{\left(\mathrm{q}_{\mathrm{k}}\right)} \cdot \mathrm{C}_{0 \mathrm{k}} \cdot \frac{\mathrm{v}_{0}}{\mathrm{~W}} \\
& (\mathrm{k}=1, \ldots, \mathrm{P}, \mathrm{P}+1, \ldots, \mathrm{Q})
\end{aligned}
$$

we rewrite Eq. 18 as follows:

$\alpha+\sum_{k=1}^{\mathrm{P}} \mathrm{m}_{\mathrm{k}} \mathrm{C}_{0 \mathrm{k}} \cdot \frac{\mathrm{V}_{0}}{\mathrm{~W}}+\mathrm{C}_{\mathrm{b}} \cdot \frac{\mathrm{V}_{0}}{\mathrm{~W}}-\left(\sum_{\mathrm{k}=\mathrm{P}+1}^{\mathrm{Q}} \mathrm{m}_{\mathrm{k}} \mathrm{C}_{0 \mathrm{k}}+\mathrm{C}_{\mathrm{a}}\right) \cdot \frac{\mathrm{V}_{0}}{\mathrm{w}}$

$$
\begin{aligned}
& -\mathrm{C} \cdot \frac{\mathrm{v}}{\mathrm{W}}+\sum_{\mathrm{k}=1}^{\mathrm{P}} \sum_{\mathrm{j}=0}^{\mathrm{q}_{\mathrm{k}}}\left(\mathrm{j}-\mathrm{n}_{\mathrm{k}}\right)\left[\mathrm{H}_{\mathrm{j}} \mathrm{L}_{(\mathrm{k})}\right]+\sum_{\mathrm{k}=\mathrm{P}+1}^{\mathrm{Q}} \sum_{\mathrm{j}=0}^{\mathrm{q}_{\mathrm{k}}}\left(\mathrm{j}-\mathrm{n}_{\mathrm{k}}\right)\left[\mathrm{H}_{\mathrm{j}} \mathrm{L}_{(\mathrm{k} k}\right]=0 \Rightarrow \\
& \alpha+\sum_{\mathrm{k}=1}^{\mathrm{P}} \mathrm{m}_{\mathrm{k}} \mathrm{C}_{0 \mathrm{k}} \cdot \frac{\mathrm{v}_{0}}{\mathrm{~W}}+\mathrm{C}_{\mathrm{b}} \cdot \frac{\mathrm{v}_{0}}{\mathrm{~W}}-\left(\sum_{\mathrm{k}=\mathrm{P}+1}^{\mathrm{Q}} \mathrm{m}_{\mathrm{k}} \mathrm{C}_{0 \mathrm{k}}+\mathrm{C}_{\mathrm{a}}\right) \cdot \frac{\mathrm{V}_{0}}{\mathrm{~W}} \\
& -\mathrm{C} \cdot \frac{\mathrm{v}}{\mathrm{W}}+\sum_{\mathrm{k}=1}^{\mathrm{P}}\left(\overline{\mathrm{n}}_{\mathrm{k}}^{\left(\mathrm{q}_{\mathrm{k}}\right)}-\mathrm{n}_{\mathrm{k}}\right) \mathrm{C}_{0 \mathrm{k}} \cdot \frac{\mathrm{V}_{0}}{\mathrm{~W}} \\
& +\sum_{\mathrm{k}=\mathrm{P}+1}^{\mathrm{Q}}\left(\overline{\mathrm{n}}_{\mathrm{k}}^{\left(\mathrm{q}_{\mathrm{k}}\right)}-\mathrm{n}_{\mathrm{k}}\right) \mathrm{C}_{0 \mathrm{k}} \cdot \frac{\mathrm{V}_{0}}{\mathrm{~W}}=0 \Rightarrow \\
& \quad \alpha+\Delta \cdot \frac{\mathrm{V}_{0}}{\mathrm{~W}}-\mathrm{C} \cdot \frac{\mathrm{V}}{\mathrm{W}}+\sum_{\mathrm{k}=1}^{\mathrm{P}}\left(\bar{n}_{\mathrm{k}}^{\left(\mathrm{q}_{\mathrm{k}}\right)}+\mathrm{m}_{\mathrm{k}}-\mathrm{n}_{\mathrm{k}}\right) \mathrm{C}_{0 \mathrm{k}} \cdot \frac{\mathrm{V}_{0}}{\mathrm{~W}} \\
& +\sum_{\mathrm{k}=\mathrm{P}+1}^{\mathrm{Q}}\left(\overline{\mathrm{n}}_{\mathrm{k}}^{\left(\mathrm{q}_{\mathrm{k}}\right)}-\mathrm{m}_{\mathrm{k}}-\mathrm{n}_{\mathrm{k}}\right) \mathrm{C}_{0 \mathrm{k}} \cdot \frac{\mathrm{V}_{0}}{\mathrm{~W}}=0
\end{aligned}
$$

Applying the identities:

$$
\begin{aligned}
& \bar{n}_{\mathrm{k}}^{\left(\mathrm{q}_{\mathrm{k}}\right)}+\mathrm{m}_{\mathrm{k}}-\mathrm{n}_{\mathrm{k}} \equiv\left(\mathrm{q}_{\mathrm{k}}+\mathrm{m}_{\mathrm{k}}-\mathrm{n}_{\mathrm{k}}\right)-\left(\mathrm{q}_{\mathrm{k}}-\overline{\mathrm{n}}_{\mathrm{k}}^{\left(\mathrm{q}_{\mathrm{k}}\right)}\right) \\
& \overline{\mathrm{n}}_{\mathrm{k}}^{\left(\mathrm{q}_{\mathrm{k}}\right)}-\mathrm{m}_{\mathrm{k}}-\mathrm{n}_{\mathrm{k}} \equiv\left(\mathrm{q}_{\mathrm{k}}-\mathrm{m}_{\mathrm{k}}-\mathrm{n}_{\mathrm{k}}\right)-\left(\mathrm{q}_{\mathrm{k}}-\overline{\mathrm{n}}_{\mathrm{k}}^{\left(\mathrm{q}_{\mathrm{k}}\right)}\right)
\end{aligned}
$$

from Eq. 21 we have, by turns,

$$
\begin{aligned}
& \alpha+\Delta \cdot \frac{\mathrm{v}_{0}}{\mathrm{~W}}-\mathrm{C} \cdot \frac{\mathrm{v}}{\mathrm{W}}+ \\
& +\sum_{\mathrm{k}=1}^{\mathrm{P}}\left(\mathrm{q}_{\mathrm{k}}+\mathrm{m}_{\mathrm{k}}-\mathrm{n}_{\mathrm{k}}\right) \mathrm{C}_{0 \mathrm{k}} \cdot \frac{\mathrm{v}_{0}}{\mathrm{~W}} \\
& -\sum_{\mathrm{k}=1}^{\mathrm{P}}\left(\mathrm{q}_{\mathrm{k}}-\overline{\mathrm{n}}_{\mathrm{k}}^{\left(\mathrm{q}_{\mathrm{k}}\right)}\right) \mathrm{C}_{0 \mathrm{k}} \cdot \frac{\mathrm{v}_{0}}{\mathrm{~W}}+ \\
& \quad \sum_{\mathrm{k}=\mathrm{P}+1}^{\mathrm{Q}}\left(\mathrm{q}_{\mathrm{k}}-\mathrm{m}_{\mathrm{k}}-\mathrm{n}_{\mathrm{k}}\right) \mathrm{C}_{0 \mathrm{k}} \cdot \frac{\mathrm{v}_{0}}{\mathrm{w}} \\
& -\sum_{\mathrm{k}=\mathrm{P}+1}^{\mathrm{Q}}\left(\mathrm{q}_{\mathrm{k}}-\overline{\mathrm{n}}_{\mathrm{k}}^{\left(\mathrm{q}_{\mathrm{k}}\right)}\right) \mathrm{C}_{0 \mathrm{k}} \cdot \frac{\mathrm{v}_{0}}{\mathrm{~W}}=0 \Rightarrow \\
& \mathrm{C} \cdot \frac{\mathrm{v}}{\mathrm{w}}=\mathrm{A} \cdot \frac{\mathrm{v}_{0}}{\mathrm{w}}+\alpha-\sum_{\mathrm{k}=1}^{\mathrm{Q}} \mathrm{G}^{\left(\mathrm{q}_{\mathrm{k}}\right)} \cdot \mathrm{C}_{0 \mathrm{k}} \cdot \frac{\mathrm{v}_{0}}{\mathrm{w}}=0 / \cdot \frac{\mathrm{w}}{\mathrm{v}_{0}} \Rightarrow \\
& \mathrm{C} \cdot \frac{\mathrm{v}}{\mathrm{v}_{0}}=\mathrm{A}+\left(1+\frac{\mathrm{v}}{\mathrm{V}_{0}}\right) \cdot \alpha-\sum_{\mathrm{k}=1}^{\mathrm{Q}} \mathrm{G}^{\left(\mathrm{q}_{\mathrm{k}}\right)} \cdot \mathrm{C}_{0 \mathrm{k}}=0 \Rightarrow
\end{aligned}
$$

where

$$
\mathrm{G}^{\left(\mathrm{q}_{\mathrm{k}}\right)}=\mathrm{q}_{\mathrm{k}}-\overline{\mathrm{n}}_{\mathrm{k}}^{\left(\mathrm{q}_{\mathrm{k}}\right)}=\sum_{\mathrm{i}=1}^{\mathrm{q}_{\mathrm{k}}} \frac{\mathrm{g}_{\mathrm{ki}}}{[\mathrm{H}]+\mathrm{g}_{\mathrm{ki}}}=\sum_{\mathrm{i}=1}^{\mathrm{q}_{\mathrm{k}}} \frac{\mathrm{g}_{\mathrm{ki}}^{*}}{\mathrm{~h}+\mathrm{g}_{\mathrm{ki}}^{*}}
$$

and alkalinity A is expressed as follows

$$
\mathrm{A}=\sum_{\mathrm{k}=1}^{\mathrm{p}}\left(\mathrm{q}_{\mathrm{k}}+\mathrm{m}_{\mathrm{k}}-\mathrm{n}_{\mathrm{k}}\right) \cdot \mathrm{C}_{0 \mathrm{k}}+\sum_{\mathrm{k}=1}^{\mathrm{Q}}\left(\mathrm{q}_{\mathrm{k}}-\mathrm{m}_{\mathrm{k}}-\mathrm{n}_{\mathrm{k}}\right) \cdot \mathrm{C}_{0 \mathrm{k}}+\Delta
$$

The $\mathrm{g}_{\mathrm{ki}}$ and $\mathrm{g}_{\mathrm{ki}}^{*}$ in Eq. 24 denote $\mathrm{i}$-th Simms constant and hybrid Simms constant related to k-th component. From Eq. 23 we get, by turns,

$$
\begin{aligned}
& (\mathrm{C}-\alpha) \cdot \frac{\mathrm{V}}{\mathrm{V}_{0}}=\mathrm{A}-\sum_{\mathrm{k}=1}^{\mathrm{Q}} \mathrm{G}^{\left(\mathrm{q}_{\mathrm{k}}\right)} \cdot \mathrm{C}_{0 \mathrm{k}}+\alpha \\
& \frac{\mathrm{V}}{\mathrm{V}_{0}}=\frac{\mathrm{A}-\sum_{\mathrm{k}=1}^{\mathrm{Q}} \sum_{\mathrm{i}=1}^{\mathrm{q}_{\mathrm{k}}} \mathrm{G}^{\left(\mathrm{q}_{\mathrm{k}}\right)} \cdot \mathrm{C}_{0 \mathrm{k}}+\alpha}{\mathrm{C}-\alpha} \\
& \frac{\mathrm{W}}{\mathrm{V}_{0}}=\frac{\mathrm{A}-\sum_{\mathrm{k}=1}^{\mathrm{Q}} \mathrm{G}^{\left(\mathrm{q}_{\mathrm{k}}\right)} \cdot \mathrm{C}_{0 \mathrm{k}}+\mathrm{C}}{\mathrm{C}-\alpha}
\end{aligned}
$$

Current concentration of $\mathrm{HB}$ from $\mathrm{T}$ in $\mathrm{D}+\mathrm{T}$ mixture, is

$$
\begin{aligned}
& c=C \cdot \frac{V}{W}=C \cdot \frac{A-\sum_{k=1}^{Q} G^{\left(q_{k}\right)} \cdot C_{0 k}+\alpha}{A-\sum_{k=1}^{Q} G^{\left(q_{k}\right)} \cdot C_{0 k}+C} \Rightarrow \\
& c=C \cdot \frac{V}{W}=C \cdot\left(1-\frac{C-\alpha}{A+C-\sum_{k=1}^{Q} G^{\left(q_{k}\right)} \cdot C_{0 k}}\right)
\end{aligned}
$$

\section{Complex D+T system (IV) - isomolar mode}

In the system IV, the charge balance (Eq. 18) and the relations:

$$
\begin{aligned}
& \sum_{j=0}^{\mathrm{q}_{\mathrm{k}}}\left[\mathrm{H}_{\mathrm{j}} \mathrm{L}_{(\mathrm{k})}\right]=\mathrm{d} \cdot \mathrm{C}_{0 \mathrm{k}}, \\
& \sum_{\mathrm{j}=0}^{\mathrm{q}_{\mathrm{k}}} \mathrm{i}\left[\mathrm{H}_{\mathrm{j}} \mathrm{L}_{(\mathrm{k})}\right]=\overline{\mathrm{n}}_{\mathrm{k}}^{\left(\mathrm{q}_{\mathrm{k}}\right)} \cdot \mathrm{d} \cdot \mathrm{C}_{0 \mathrm{k}}(\mathrm{k}=1, \ldots, \mathrm{P}, \mathrm{P}+1, \ldots, \mathrm{Q}) ; \\
& {[\mathrm{K}]=\sum_{\mathrm{k}=1}^{\mathrm{P}} \mathrm{m}_{\mathrm{k}} \cdot \mathrm{d} \cdot \mathrm{C}_{0 \mathrm{k}} ;} \\
& {[\mathrm{M}]=\mathrm{d} \cdot \mathrm{C}_{\mathrm{b}}+\frac{\mathrm{C}_{1} \mathrm{~V}_{\mathrm{MB}}}{\mathrm{V}_{\mathrm{f}}}-\frac{\mathrm{CV}_{\mathrm{B}}}{\mathrm{V}_{\mathrm{f}}} \cdot \frac{\mathrm{V}}{\mathrm{W}} ;} \\
& {[\mathrm{B}]=\mathrm{d} \cdot\left(\sum_{\mathrm{k}=\mathrm{P}+1}^{\mathrm{Q}} \mathrm{m}_{\mathrm{k}} \cdot \mathrm{C}_{0 \mathrm{k}}+\mathrm{C}_{\mathrm{a}}\right)+\frac{\mathrm{C}_{1} \mathrm{~V}_{\mathrm{MB}}}{\mathrm{V}_{\mathrm{f}}}}
\end{aligned}
$$

are valid, see Eq. 12. Putting Equations 27 in Eq. 18 gives

$$
\begin{aligned}
& \alpha+\mathrm{d} \cdot \sum_{\mathrm{k}=1}^{\mathrm{P}} \mathrm{m}_{\mathrm{k}} \cdot \mathrm{C}_{0 \mathrm{k}}+\mathrm{d} \cdot \mathrm{C}_{\mathrm{b}}-\frac{\mathrm{CV}_{\mathrm{B}}}{\mathrm{V}_{\mathrm{f}}} \cdot \frac{\mathrm{v}}{\mathrm{W}}- \\
& \mathrm{d} \cdot\left(\sum_{\mathrm{k}=\mathrm{P}+1}^{\mathrm{Q}} \mathrm{m}_{\mathrm{k}} \cdot \mathrm{C}_{0 \mathrm{k}}+\mathrm{C}_{\mathrm{a}}\right)+ \\
& \mathrm{d} \cdot \sum_{\mathrm{k}=1}^{\mathrm{Q}}\left(\overline{\mathrm{n}}_{\mathrm{k}}^{\left(\mathrm{q}_{\mathrm{k}}\right)}-\mathrm{n}_{\mathrm{k}}\right) \cdot \mathrm{C}_{0 \mathrm{k}}=0 \Rightarrow \\
& \alpha+\mathrm{d} \cdot \Delta-\frac{\mathrm{CV} \mathrm{B}}{\mathrm{V}_{\mathrm{f}}} \cdot \frac{\mathrm{v}}{\mathrm{W}} \\
& +\mathrm{d} \cdot \sum_{\mathrm{k}=1}^{\mathrm{P}}\left(\overline{\mathrm{n}}_{\mathrm{k}}^{\left(\mathrm{q}_{\mathrm{k}}\right)}-\mathrm{n}_{\mathrm{k}}+\mathrm{m}_{\mathrm{k}}\right) \cdot \mathrm{C}_{0 \mathrm{k}}+
\end{aligned}
$$




$$
\begin{aligned}
& \mathrm{d} \cdot \sum_{\mathrm{k}=\mathrm{P}+1}^{\mathrm{Q}}\left(\overline{\mathrm{n}}_{\mathrm{k}}^{\left(\mathrm{q}_{\mathrm{k}}\right)}-\mathrm{n}_{\mathrm{k}}-\mathrm{m}_{\mathrm{k}}\right) \cdot \mathrm{C}_{0 \mathrm{k}}=0 \Rightarrow \\
& \frac{\mathrm{CV}_{\mathrm{B}}}{\mathrm{V}_{\mathrm{f}}} \cdot \frac{\mathrm{v}}{\mathrm{W}}=\alpha+\mathrm{d} \cdot \Delta+\mathrm{d} \cdot \sum_{\mathrm{k}=1}^{\mathrm{P}}\left(\overline{\mathrm{n}}_{\mathrm{k}}^{\left(\mathrm{q}_{\mathrm{k}}\right)}-\mathrm{n}_{\mathrm{k}}+\mathrm{m}_{\mathrm{k}}\right) \cdot \mathrm{C}_{0 \mathrm{k}} \\
& +\mathrm{d} \cdot \sum_{\mathrm{k}=\mathrm{P}+1}^{\mathrm{Q}}\left(\overline{\mathrm{n}}_{\mathrm{k}}^{\left(\mathrm{q}_{\mathrm{k}}\right)}-\mathrm{n}_{\mathrm{k}}-\mathrm{m}_{\mathrm{k}}\right) \cdot \mathrm{C}_{0 \mathrm{k}} \Rightarrow \\
& \mathrm{c}=\frac{\mathrm{CV}_{\mathrm{B}}}{\mathrm{V}_{\mathrm{f}}} \cdot \frac{\mathrm{v}}{\mathrm{W}}=\mathrm{d} \cdot \mathrm{A}+\alpha-\mathrm{d} \cdot \sum_{\mathrm{k}=1}^{\mathrm{Q}} \mathrm{G}^{\left(\mathrm{q}_{\mathrm{k}}\right)} \cdot \mathrm{C}_{0 \mathrm{k}}
\end{aligned}
$$

where A is identical with Eq. 25; see also the remark at Eq. 25, as a particular case of Eq. 28.

\section{Dynamic buffer capacity}

The c values (Equations 11, 17, 26, 28), expressing current concentration of $\mathrm{HB}$ (C) from $\mathrm{T}$ in $\mathrm{D}+\mathrm{T}$ mixture, are the basis to formulate the dynamic buffer capacity

$$
\beta_{\mathrm{V}}=\left|\frac{\mathrm{dc}}{\mathrm{dph}}\right|
$$

Recapitulating, for normal mode (systems I and III), we have $\mathrm{c}=\mathrm{CV} / \mathrm{W}$, and for isomolar mode (systems II and IV) we have $\mathrm{c}=\mathrm{C}_{\mathrm{B}} \cdot \frac{\mathrm{V}}{\mathrm{W}}$, where $\mathrm{C}_{\mathrm{B}}=$ $\frac{\mathrm{CV}_{\mathrm{B}}}{\mathrm{V}_{\mathrm{f}}}$.

Applying the general relation

$\frac{\mathrm{dz}}{\mathrm{dph}}=\frac{\mathrm{dz}}{\mathrm{dh}} \cdot \frac{\mathrm{dh}}{\mathrm{dph}}=-\ln 10 \cdot \mathrm{h} \cdot \frac{\mathrm{dz}}{\mathrm{dh}}$

- $\quad$ to $\mathrm{z}=\alpha$ (Eq. 3), we have

$\frac{\mathrm{d} \alpha}{\mathrm{dh}}=\frac{1}{\gamma}+\mathrm{K}_{\mathrm{W}} \gamma / \mathrm{h}^{2}$

$\mathrm{h} \cdot \frac{\mathrm{d} \alpha}{\mathrm{dh}}=\frac{\mathrm{h}}{\gamma}+\mathrm{K}_{\mathrm{W}} \gamma / \mathrm{h}=[\mathrm{H}]+[\mathrm{OH}]$

$\frac{\mathrm{d} \alpha}{\mathrm{dph}}=-\ln 10 \cdot([\mathrm{H}]+[\mathrm{OH}])$

- to $\mathrm{z}=\mathrm{G}^{\text {(q) }}$ (Eq. 9), we have

$\mathrm{h} \cdot \frac{\mathrm{dG}^{(\mathrm{q})}}{\mathrm{dh}}=-\sum_{\mathrm{i}=1}^{\mathrm{q}} \frac{\mathrm{h} \cdot \mathrm{g}_{\mathrm{i}}^{*}}{\left(\mathrm{~h}+\mathrm{g}_{\mathrm{i}}^{*}\right)^{2}}=-\sum_{\mathrm{i}=1}^{\mathrm{q}} \frac{[\mathrm{H}] \cdot \mathrm{g}_{\mathrm{i}}}{\left([\mathrm{H}]+\mathrm{g}_{\mathrm{i}}\right)^{2}}$

we obtain the formulas for $\beta_{\mathrm{V}}$ related to particular systems (I) - (IV). Note that $[\mathrm{H}]+[\mathrm{OH}]=\left(\alpha^{2}+4 \mathrm{~K}_{\mathrm{W}}\right)^{1 / 2}$, $\mathrm{K}_{\mathrm{W}}=[\mathrm{H}][\mathrm{OH}]$, see Equations 3 and 31.

\section{The $\beta_{\mathrm{V}}$ for the system (I)}

From Eq. 11 we obtain

$$
\begin{aligned}
& h \cdot \frac{d c}{d h}=C \cdot \frac{h \cdot \frac{d \alpha}{d h} \cdot\left(A+C-G^{(q)} \cdot C_{0}\right)-(C-\alpha) \cdot C_{0} \cdot h \cdot \frac{d G(q)}{d h}}{\left(A+C-C_{0} \cdot G_{q}\right)^{2}} \Rightarrow \\
& \beta_{\mathrm{V}}=\left|\frac{\mathrm{dc}}{\mathrm{dph}}\right|=\ln 10 \cdot \mathrm{C} . \\
& \frac{([\mathrm{H}]+[\mathrm{OH}]) \cdot\left(\mathrm{A}+\mathrm{C}-\mathrm{C}_{0} \cdot \sum_{\mathrm{i}=1}^{\mathrm{q}} \frac{\mathrm{g}_{\mathrm{i}}}{[\mathrm{H}]+\mathrm{g}_{\mathrm{i}}}\right)+(\mathrm{C}-\alpha) \cdot \mathrm{C}_{0} \cdot \sum_{\mathrm{i}=1}^{\mathrm{q}} \frac{[\mathrm{H}] \cdot \mathrm{g}_{\mathrm{i}}}{\left([\mathrm{H}]+\mathrm{g}_{\mathrm{i}}\right)^{2}}}{\left(\mathrm{~A}+\mathrm{C}-\mathrm{C}_{0} \cdot \sum_{\mathrm{i}=1}^{\mathrm{q}} \frac{\mathrm{gi}_{\mathrm{i}}}{[\mathrm{H}]+\mathrm{g}_{\mathrm{i}}}\right)^{2}}
\end{aligned}
$$

As we see, the $\beta_{V}$ is expressed in terms of $[\mathrm{H}]$ and $g_{i}$.

\section{The $\beta_{\mathrm{V}}$ for the system (III)}

From Eq. 26 we have

$$
\begin{aligned}
& \beta_{\mathrm{V}}=\left|\frac{\mathrm{dc}}{\mathrm{dph}}\right|=\ln 10 \cdot \mathrm{C} \cdot
\end{aligned}
$$

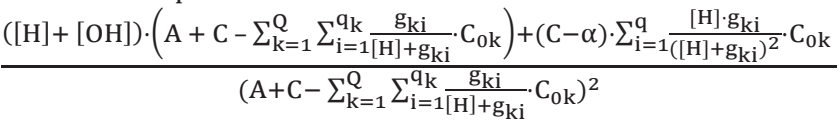

\section{The $\beta_{V}$ for the system (II)}

From Eq. 17 we have

$$
\begin{aligned}
& h \cdot \frac{d c}{d h}=h \cdot \frac{d \alpha}{d h}-d \cdot h \cdot \frac{d G^{(q)}}{d h} \cdot C_{0} \\
& \beta_{V}=\left|\frac{d c}{d p h}\right|=\ln 10 \cdot\left(([H]+[O H])+d \cdot C_{0} \cdot \sum_{i=1}^{q} \frac{[H] \cdot g_{i}}{\left([H]+g_{i}\right)^{2}}\right)
\end{aligned}
$$

\section{The $\beta_{\mathrm{V}}$ for the system (IV)}

From Eq. 28 we have

$$
\beta_{\mathrm{V}}=\left|\frac{\mathrm{dc}}{\mathrm{dph}}\right|=\ln 10 \cdot\left(([\mathrm{H}]+[\mathrm{OH}])+\mathrm{d} \cdot \sum_{\mathrm{k}=1}^{\mathrm{Q}} \sum_{\mathrm{i}=1}^{\mathrm{q}} \frac{\left[\mathrm{H} \cdot \mathrm{g}_{\mathrm{gi}}\right.}{\left.(\mathrm{H}]+\mathrm{g}_{\mathrm{ki}}\right)^{2}} \cdot \mathrm{C}_{0 \mathrm{k}}\right)
$$

From Equations (35) and (36) it results that $\beta_{V}$ does not depend on alkalinity $A$ when the titration is realized according to isomolar mode. The $\beta_{\mathrm{V}}$ depends on $\mathrm{A}$ when the titration is performed according to normal mode, see Equations 33 and 34.

\section{Simms constants in a system with hydroxo- complexes}

The Simms constants can be also related to the system of hydroxo-complexes, $\mathrm{Me}(\mathrm{OH})_{\mathrm{i}}{ }^{+n-\mathrm{i}}(\mathrm{i}=1, \ldots, \mathrm{p})$ formed by hydrolysable cations. Assuming $p=2$, we define 


$$
\overline{\mathrm{n}}^{(2)}=\frac{[\mathrm{MeOH}]+2\left[\mathrm{Me}(\mathrm{OH})_{2}\right]}{[\mathrm{Me}]+[\mathrm{MeOH}]+\left[\mathrm{Me}(\mathrm{OH})_{2}\right]}
$$

Applying the stability constants $\mathrm{K}_{\mathrm{i}} \mathrm{OH}$ of hydroxocomplexes

$$
\mathrm{Me}^{+\mathrm{u}}+\mathrm{iOH}^{-1}=\mathrm{Me}(\mathrm{OH})_{\mathrm{i}}{ }^{+\mathrm{u}-\mathrm{i}} \Rightarrow \mathrm{K}_{\mathrm{i}}^{\mathrm{OH}}=\frac{\left[\mathrm{Me}(\mathrm{OH})_{\mathrm{i}}\right]}{[\mathrm{Me}][\mathrm{OH}]^{\mathrm{i}}}
$$

we have, in particular,

$$
\overline{\mathrm{n}}^{(2)}=\frac{\mathrm{K}_{1}{ }^{\mathrm{OH}}[\mathrm{OH}]+2 \mathrm{~K}_{1}{ }^{\mathrm{OH}}[\mathrm{OH}]^{2}}{1+\mathrm{K}_{1}{ }^{\mathrm{OH}}[\mathrm{OH}]+\mathrm{K}_{1}{ }^{\mathrm{OH}}[\mathrm{OH}]^{2}}=\frac{\mathrm{K}_{1}{ }^{\mathrm{OH}} \mathrm{K}_{\mathrm{W}}[\mathrm{H}]+2 \mathrm{~K}_{1}{ }^{\mathrm{OH}} \mathrm{K}_{\mathrm{W}}{ }^{2}}{[\mathrm{H}]^{2}+\mathrm{K}_{1}{ }^{\mathrm{OH}} \mathrm{K}_{\mathrm{W}}[\mathrm{H}]+\mathrm{K}_{1}{ }^{\mathrm{OH}} \mathrm{K}_{\mathrm{W}}{ }^{2}}
$$

where $\mathrm{K}_{W}=[\mathrm{H}][\mathrm{OH}]$. The right side of Eq. 39 can be presented as the sum of two partial fractions

$$
\overline{\mathrm{n}}^{(2)}=\frac{\mathrm{g}_{1}}{[\mathrm{H}]+\mathrm{g}_{1}}+\frac{\mathrm{g}_{2}}{[\mathrm{H}]+\mathrm{g}_{2}}=\frac{\left(\mathrm{g}_{1}+\mathrm{g}_{2}\right)[\mathrm{H}]+2 \mathrm{~g}_{1} \mathrm{~g}_{2}}{[\mathrm{H}]^{2}+\left(\mathrm{g}_{1}+\mathrm{g}_{2}\right)[\mathrm{H}]+\mathrm{g}_{1} \mathrm{~g}_{2}}
$$

Comparing the right sides of Equations 39 and 40, we get

$$
\mathrm{g}_{1}+\mathrm{g}_{2}=\mathrm{K}_{1}{ }^{\mathrm{OH}} \mathrm{K}_{\mathrm{W}} \text { and } \mathrm{g}_{1} \cdot \mathrm{g}_{2}=\mathrm{K}_{2}{ }^{\mathrm{OH}} \mathrm{K}_{\mathrm{W}}{ }^{2}
$$

Comparing the Equations 41 and Equation 9 obtained for $\mathrm{q}=2$, we see obvious similarities between $2-\overline{\mathrm{n}}^{(2)}$ for proto-complexes and $\overline{\mathrm{n}}^{(2)}$ for hydroxocomplexes $(\mathrm{p}=2)$. Analogous regularities are valid also for other $\mathrm{p}=\mathrm{q}$ values.

\section{Calculation of $g_{i}$ values}

The $\mathrm{g}_{\mathrm{i}}$ values can be calculated under assumption that $\mathrm{K}_{\mathrm{i}}^{\mathrm{H}}$ or $\mathrm{K}_{\mathrm{i}}$ values are known beforehand. Such calculations can be made with use of the iterative computer program, exemplified in [14] for $q=4$. In particular, for DCTA $=\mathrm{H}_{4} \mathrm{~L}(\mathrm{q}=4)$ we have [21]: $\log \mathrm{K}_{1}{ }^{\mathrm{H}}$ $=11.78, \log \mathrm{K}_{2} \mathrm{H}=17.98, \log \mathrm{K}_{3} \mathrm{H}=21.58, \log \mathrm{K}_{4} \mathrm{H}=24.09$, i.e., $\mathrm{pK}_{1}=2.51, \mathrm{pK}_{2}=3.60, \mathrm{pK}_{3}=6.20, \mathrm{pK}_{4}=11.78$, and then we get: $\mathrm{pg}_{1}=2.550589, \mathrm{pg}_{2}=3.560505, \mathrm{pg}_{3}=$ 6.198906, $\mathrm{pg}_{4}=11.779999$. As we see, the $\mathrm{pg}_{\mathrm{i}}$ values are not distant from $\mathrm{pK} \mathrm{K}_{\mathrm{i}}$ values and then $\mathrm{pK}_{\mathrm{i}}$ values can be taken as starting values for $\mathrm{pg}_{\mathrm{i}}$, when calculations are made according to an iterative procedure, e.g. one offered by MATLAB [22]. When the successive $\mathrm{pK}_{\mathrm{i}}$ values are more distant, the $\mathrm{pg}_{\mathrm{i}}$ values are very close to the $\mathrm{pK}_{\mathrm{i}}$ values, $\mathrm{pg}_{\mathrm{i}} \approx \mathrm{pK}_{\mathrm{i}}$. Such a case occurs e.g. for $\mathrm{H}_{2} \mathrm{CO}_{3}(\mathrm{q}=2): \mathrm{pK}_{1}=6.3, \mathrm{pK}_{2}=10.1$, where $\mathrm{pg}_{1}=$ 10.099931, $\mathrm{pg}_{2}=6.300069$. Generally, the differences $\left|p K_{i}-p_{i}\right|$ are more distinct when the successive $\mathrm{pK}_{\mathrm{i}}$ values for an acid are closer to each other.

\section{Interrelations between $K_{i}$ and $g_{i}$ values for a $q-$ protic acid $\mathrm{H}_{\mathrm{n}} \mathrm{L}$}

Generalizing and applying the summation notations, we get [14]

$$
\begin{aligned}
& \mathrm{K}_{1}=\sum_{\mathrm{i}=1}^{\mathrm{q}} \mathrm{g}_{\mathrm{i}}, \mathrm{K}_{1} \cdot \mathrm{K}_{2}=\sum_{\mathrm{i}=1}^{\mathrm{q}-1} \sum_{\mathrm{j}=\mathrm{i}+1}^{\mathrm{q}} \mathrm{g}_{\mathrm{i}} \cdot \mathrm{g}_{\mathrm{j}}, \\
& \mathrm{K}_{1} \cdot \mathrm{K}_{2} \cdot \mathrm{K}_{3}=\sum_{\mathrm{i}=1}^{\mathrm{q}-2} \sum_{\mathrm{j}=\mathrm{i}+1}^{\mathrm{q}-1} \sum_{\mathrm{k}=\mathrm{j}+1}^{\mathrm{q}} \mathrm{g}_{\mathrm{i}} \cdot \mathrm{g}_{\mathrm{j}} \cdot \mathrm{g}_{\mathrm{k}}, \\
& \ldots, \mathrm{K}_{1} \cdot \mathrm{K}_{2} \cdot \ldots \cdot \mathrm{K}_{\mathrm{q}}=\mathrm{g}_{1} \cdot \mathrm{g}_{2} \cdot \ldots \cdot \mathrm{g}_{\mathrm{q}}
\end{aligned}
$$

The expression for $\prod_{i=1}^{k} K_{i}(E q, 22)$, formulated for q-protic acid, is a sum involving

$$
\left(\begin{array}{l}
\mathrm{q} \\
\mathrm{k}
\end{array}\right)=\frac{\mathrm{q} !}{\mathrm{k} !(\mathrm{q}-\mathrm{k}) !}
$$

components formed from $\mathrm{k}$ different $\mathrm{g}_{\mathrm{i}}$ values. In combinatorics, the binomial coefficient $\left(\begin{array}{l}\mathrm{q} \\ \mathrm{k}\end{array}\right)$ [23] expresses the number of distinct k-element subsets (combinations) formed from a set containing $q$ different elements [24], as in the Pascal's triangle [25]. For further details see $[14,26]$.

\section{Calculation of alkalinity (A) from equation for titration curve}

Example. $\mathrm{V}_{0} \mathrm{~mL}$ of $\mathrm{NaHCO}_{3}\left(\mathrm{C}_{01}\right)+\mathrm{Na}_{2} \mathrm{CO}_{3}\left(\mathrm{C}_{02}\right)$ solution as $\mathrm{D}$ is titrated with $\mathrm{V} \mathrm{mL}$ of $\mathrm{HCl}(\mathrm{C})$ as $\mathrm{T}$. The desired form of equation for titration curve is derived as follows:

$$
\begin{aligned}
& \alpha+\left(\mathrm{C}_{01}+2 \mathrm{C}_{02}\right) \cdot \frac{\mathrm{V}_{0}}{\mathrm{~W}}-\mathrm{C} \cdot \frac{\mathrm{V}}{\mathrm{W}}=\left(2-\overline{\mathrm{n}}^{(2)}\right) \cdot\left(\mathrm{C}_{01}+\mathrm{C}_{02}\right) \\
& \mathrm{C} \cdot \frac{\mathrm{V}}{\mathrm{W}}=\left(\mathrm{C}_{01}+2 \mathrm{C}_{02}\right) \cdot \frac{\mathrm{V}_{0}}{\mathrm{~W}}+\alpha-\left(2-\overline{\mathrm{n}}^{(2)}\right) \cdot\left(\mathrm{C}_{01}+\mathrm{C}_{02}\right) \cdot \frac{\mathrm{V}_{0}}{\mathrm{~W}} \\
& \mathrm{C} \cdot \frac{\mathrm{V}}{\mathrm{V}_{0}}=\left(\mathrm{C}_{01}+2 \mathrm{C}_{02}\right)+\left(1+\frac{\mathrm{V}}{\mathrm{V}_{0}}\right) \cdot \alpha-\left(\sum_{\mathrm{k}=1}^{2} \frac{\mathrm{g}_{\mathrm{k}}}{[\mathrm{H}]+\mathrm{g}_{\mathrm{k}}}\right) \cdot\left(\mathrm{C}_{01}+\mathrm{C}_{02}\right)
\end{aligned}
$$

where $\mathrm{A}=\mathrm{C}_{01}+2 \mathrm{C}_{02}$, see Eq. 23 .

\section{Acid-Base Microequilibria as a Stochastic Process}

On the basis of formulation with the Simms constants involved one can state that the dissociation of $\mathrm{H}^{+1}$ from different protonation sites/centers proceeds independently, and the proton uptake/dissociation from/to these sites (basicity centers) can be perceived as a stochastic process, categorized in terms of a success/failure. The degree of dissociation $\mathrm{HL}_{(\mathrm{i})}=\mathrm{H}^{+1}+\mathrm{L}_{(\mathrm{i}) 1^{-1}}$ from the $\mathrm{i}$-th site is 


$$
\alpha_{\mathrm{i}}=\alpha_{\mathrm{i}}(\mathrm{pH})=\frac{1}{10^{\mathrm{pg} \mathrm{i}_{\mathrm{i}}-\mathrm{pH}}+1}=\frac{1}{\mathrm{e}^{\beta \cdot\left(\mathrm{pg}_{\mathrm{i}}-\mathrm{pH}\right)}+1}
$$

where $\beta=\ln 10$. The $\alpha_{i}=\alpha_{i}(\mathrm{pH})$ fulfills the properties of cumulative distribution function

$$
\begin{aligned}
& \lim _{\mathrm{pH} \rightarrow-\infty} \alpha_{\mathrm{i}}(\mathrm{pH})=0 \\
& \lim _{\mathrm{pH} \rightarrow \infty} \alpha_{\mathrm{i}}(\mathrm{pH})=1 \\
& \alpha_{\mathrm{i}}(\mathrm{pH})=\int_{-\infty}^{\mathrm{pH}} \mathrm{f}_{\mathrm{i}}(\mathrm{x}) \cdot \mathrm{dx}
\end{aligned}
$$

where $y=f_{i}(p H)$ is the probability density function

$$
\mathrm{f}_{\mathrm{i}}(\mathrm{pH})=\frac{\mathrm{d} \alpha_{\mathrm{i}}(\mathrm{pH})}{\mathrm{dpH}}
$$

It implies that

$$
f_{i}(p H)=\beta \cdot \frac{10^{\mathrm{pg}_{i}-p H}}{\left(10^{\mathrm{pg}_{\mathrm{i}}-\mathrm{pH}^{2}}+1\right)^{2}}=\beta \cdot \alpha_{\mathrm{i}}(\mathrm{pH}) \cdot\left(1-\alpha_{\mathrm{i}}(\mathrm{pH})\right)
$$

The function plotted in Figure 1 appears some similarities with the Fermi-Dirac distribution function [27].

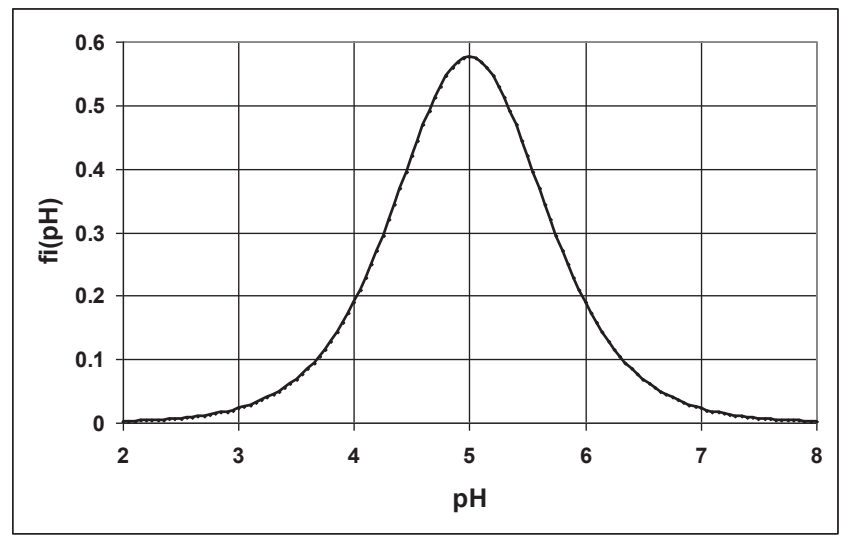

Figure 1: The function $y=f_{i}(p H)$ (Eq. 20) plotted for $p g_{i}=5.0$.

\section{A link between the species of defined and undefined composition}

In order to assume a "common basis" between species of defined composition and ones of undefined composition, such as fulvic acids [14], a formal procedure of their "shredding" into virtual monoprotic acids can be used. Each protongenerating or proton-accepting place of the resulting monoprotic acids or their bases will be considered as the center of acidity or basicity. The approach based on application of Simms constants meets these aspirations. Generally, any of the monoprotic acids ascribed to the polyprotic acid of a defined composition, will be an acid with undefined composition (and thus an undefined molecular mass), like a monoprotic acid, resulting from similar, mental operation applied to fulvic acid molecules. The difference is that, in the first case, the concentrations of the virtual monoprotic acids are known, and equal to the concentration of the generic, polyprotic acid.

Most bio-and drug molecules contain two or more basic sites. Their properties, in particular, charge and charge distribution within their structure are characterized by various acid-base parameters, e.g. protonation constant ( $\mathrm{pK}$ ). In ${ }^{1} \mathrm{H}$ NMR method, $\mathrm{pH}$ can be calculated from chemical shifts of indicator molecules throughout the pH-scale (0-14). A subsequent aim was to accurately determine the microscopic protonation constants of the most basic natural amino acid, arginine, by ${ }^{1} \mathrm{H}$ NMR-pH titrations. Developing a ${ }^{15} \mathrm{~N}$ NMR method for the determination of group-specific basicities of individual amino groups. The basicity centers can be also referred to micro-constants [28], related to bioanalysis, drug metabolism and pharmacokinetic analysis of small molecules, macromolecules, tested with use of LC/MS/MS, HPLC/UV, and immunoassay techniques [29].

The fate of ionizable drug molecules in the body depends on external parameters, especially $\mathrm{pH}$ in extramolecular environment. Binding of drug molecules to the target is highly affected by the protonation state of ionizable groups. Site-specific basicities can be quantified by the microscopic protonation constants, which provide information on their submolecular basicity. Regardless of a concrete dissociation scheme, the assumption about independent dissociation for each ionogenic group leads to a one-to-one correspondence between the sets of intrinsic (microscopic) and apparent (macroscopic) constants. The assumption of independent dissociation enormously simplifies the theoretical treatment.

To interrelate microscopic acidity constants (pK values) of polyprotic acids or bases with their macroscopic pK values, the Ising models [30], which have received much attention in statistical mechanics, were used in [31].

Consideration of molar concentration of some macromolecules, such as HAs and FAs is impossible, owing to undefined molecular and then molar masses of these acids. One can only define approximate molar concentrations of acidity/basicity centers (mainly carboxylic acids and primary amine groups) in these acids and then recalculate them on the total mass or volume of the sample tested. 
The equilibrium constant values at high ionic strengths may differ significantly from those obtained in more diluted solutions. It should be noted that the equilibrium constants cited e.g. in [21], come from the papers published over many decades. Earlier equilibrium constants were determined using models adapted to contemporary computing capabilities.

Normal mode of acid-base titration in the D+T system presented above is usually associated with a significant change in ionic strength of the solution, which changes the activity coefficient $(\gamma)$, and consequently the equilibrium constants $\left(\mathrm{K}_{\mathrm{i}}\right)$ values [13]. It was the main leitmotif of application of titration according to isomolar instead of normal mode, as indicated above.

\section{Final comments}

The novel approach to acid-base equilibria, based on application of Simms constants idea, is derived on the basis of charge and concentration balances, and applied - for illustrative purposes - to titrations in $\mathrm{D}+\mathrm{T}$ systems of different complexity. This approach was suggested as one applicable to formulation of total alkalinity (TAL) in natural waters $[12,13]$, also with fulvic acids [14], and their complexes with different metal ions. This idea can be extended on biological systems, such as lymphatic systems in living organisms, as well. The models are perceived as the challenge/counterproposal put towards the approaches known hithertpo in the literature.

\section{References}

1. Simms HS (1926) Dissociation of polyvalent substances. I. Relation of constants to titration data. J Am Chem Soc 48: 1239-1250. Link: http://bit.ly/2Sjk2b5

2. Simms HS (1926) Dissociation of polyvalent substances. II. Relation of constants to chemical structure. J Am Chem Soc 48: 1251-1261. Link: http://bit.ly/38mhiPJ

3. Michałowski T (1992) Some new algorithms applicable to potentiometric titration in acid-base systems. Talanta 39: 1127-1137. Link: http://bit.ly/2SjOamL

4. Michałowski T, Gibas E (1994) Applicability of new algorithms for determination of acids, bases, salts and their mixtures. Talanta 41: 13111317. Link: http://bit.ly/39p7CUX

5. Michałowski T, Rokosz A, Tomsia A (1987) Determination of basic impurities in mixture of hydrolysable salts. Analyst 112: 1739-1741. Link: https://rsc.li/2vshePS

6. Michałowski T (1988) Possibilities of application of some new algorithms for standardization purposes; Standardisation of sodium hydroxide solution against commercial potassium hydrogen phthalate. Analyst 113: 833-935. Link: http://bit.ly/39yyrGp
7. Michałowski T, Rokosz A, Negrusz-Szczęsna E (1988) Use of Padé approximants in the processing of $\mathrm{pH}$ titration data; Determination of the parameters involved in the titration of acetic acid. Analyst 113: 969972. Link: https://rsc.li/2SzbprU

8. Michałowski T, Rokosz A, Kościelniak P, Łagan JM, Mrozek J (1989) Calculation of concentrations of hydrochloric and citric acids together in mixture with hydrolysable salts. Analyst 114: 1689-1692. Link: http://bit.ly/38lwSLE

9. Janecki D, Styszko-Grochowiak K, Michałowski T (2000) The Catenation and Isomerisation Effects on Stability Constants of Complexes Formed by Some Diprotic Acids. Talanta 52: 555-562. Link: http://bit.ly/2SkrpiM

10. Janecki D, Doktór K, Michałowski T (1999) Determination of stability constants of complexes of MiKjHkL type in concentrated solutions of mixed salts. Talanta 48: 1191-1197. Link: http://bit.ly/2ONAnCl

11. Janecki D, Michałowski T (1999) Evaluation of equilibrium constants - a new approach. Chemia Analityczna (Warsaw) 44: 611-621. Link: http://bit.ly/2uvd8GV

12. Asuero AG, Michałowski T (2011) Comprehensive formulation of titration curves referred to complex acid-base systems and its analytical implications. Crit Rev Anal Chem 41: 151-187. Link: http://bit.ly/2SBJw2y

13. Michałowski T, Asuero AG (2012) New approaches in modelling the carbonate alkalinity and total alkalinity. Crit Rev Anal Chem 42: 220-244. Link: http://bit.ly/2vnexiG

14. Michałowska-Kaczmarczyk AM, Michałowski T (2016) Application of Simms Constants in Modelling the Titrimetric Analyses of Fulvic Acids and Their Complexes with Metal Ions. J Solution Chem 45: 200-220. Link: http://bit.ly/2NRXBto

15. Michałowska-Kaczmarczyk AM, Spórna-Kucab A, Michałowski T, Asuero AG (2018) The Simms constants as parameters in hyperbolic functions related to acid-base titration curves. Journal of Chemistry and Applied Chemical Engineering 2: 1. Link: http://bit.ly/31MQQwd

16. Partial fraction decomposition. Link: http://bit.ly/2P2y2UT

17. Irreducible polynomial. Link: http://bit.ly/2ShT3Nc

18. Michałowska-Kaczmarczyk AM, Michałowski T (2015) Dynamic Buffer Capacity in Acid-Base Systems. J Solution Chem 44: 1256-1266. Link: http://bit.ly/2uDNlw4

19. Michałowska-Kaczmarczyk AM, Spórna-Kucab A, Michałowski T (2017) Dynamic Buffer Capacities in Redox Systems. Biochem Mol Biol J 3: 11. Link: http://bit.ly/37n9Gv4

20. Michałowska-Kaczmarczyk AM, Spórna-Kucab A, Michałowski T (2017) Dynamic Buffer Capacities in Redox Systems. J Chem Appl Chem Eng 1: 2. Link: http://bit.ly/2SipZ8k

21. Inczédy J (1976) Analytical Applications of Complex Equilibria; Ellis Horwood: Chichester, UK. Link: http://bit.ly/2uszCs1

22. Michałowski T (2011) Application of GATES and MATLAB for Resolution of Equilibrium. Metastable and Non-Equilibrium Electrolytic Systems. In: Applications of MATLAB in Science and Engineering (ed. by $T$. Michałowski), InTech - Open Access publisher in the fields of Science, Technology and Medicine 1: 1-34. Link: http://bit.ly/3bv7xRf

23. Binomial coefficient. Link: http://bit.ly/39rK41z 
24. Combinatorics. Link: http://bit.ly/31JZFqP

25. Pascal's triangle. Link: http://bit.ly/39rKfdf

26. Michałowska-Kaczmarczyk AM, Spórna-Kucab A, Michałowski T, Asuero AG (2018) The Simms constants as parameters in hyperbolic functions related to acid-base titration curves. Journal of Chemistry and Applied Chemical Engineering 2: 1-5. Link: http://bit.ly/31MQQwd

27. Derivation of the Fermi-Dirac distribution function. Link: http://bit.ly/31N063r
28. MicroConstants. Link: http://bit.ly/20MGN58

29. Takács-Novák K, Avdeef A, Box, KJ, Podányi B, Szász G (1994) Determination of protonation macro- and microconstants and octanol/ water partition coefficient of the antiinflammatory drug niflumic acid. $J$ Pharm Biomed Anal 12: 1369-1377. Link: http://bit.ly/37nj3ee

30. Ising E (1925) Beitrag zur Theorie des Ferromagnetismus. Zeitschrift für Physik 31: 253-258. Link: http://bit.ly/2vmGKpZ

31. Borkovec M, Koper G (1994) Ising Models of Polyprotic Acids and Bases. J Phys Chem 98: 6038-6045. Link: http://bit.ly/2tPIxDo
Discover a bigger Lepact and Visibility of your artiche patieation with Peertechz Publications

\section{Highlights}

- Sgnalsry publinef of Oincid

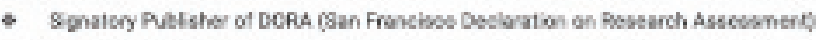

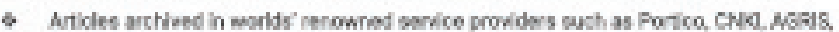

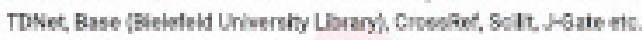

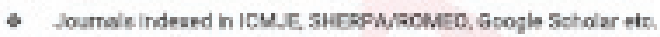

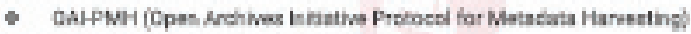

* Dedteated Eshorisi Baard foe every jouma

- Acturate and rapid peer review process

- hereasad etebions of putlishad articles through pronobisns

- Reduoed tmelhe for article pibliostion

Sutmi pour artioles and exparlance a now arge in patikation services

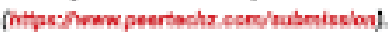

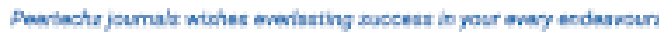

Copyright: () 2020 Michałowska-Kaczmarczyk AM, et al. This is an open-access article distributed under the terms of the Creative Commons Attribution License, which permits unrestricted use, distribution, and r eproduction in any medium, provided the original author and source are credited. 